\section{Cahiers balkaniques}

36-37 | 2008

L'image de la période ottomane dans les littératures balkaniques

\title{
Écriture, histoire et action
}

Comment lire Le Derviche et la mort de Meša Selimović ?

Writing, History, and Action: How to Read "Death and the Dervish" by Meša

Selimović?

\section{Branka Šarančić}

\section{Q OpenEdition}

\section{Journals}

Édition électronique

URL : https://journals.openedition.org/ceb/1484

DOI : $10.4000 / c e b .1484$

ISSN : 2261-4184

Éditeur

INALCO

\section{Édition imprimée}

Date de publication : 1 décembre 2008

Pagination : $61-78$

ISBN : 978-2-85831-173-6

ISSN : 0290-7402

Référence électronique

Branka Šarančić, « Écriture, histoire et action », Cahiers balkaniques [En ligne], 36-37 | 2008, mis en ligne le 15 mai 2012, consulté le 06 juillet 2021. URL : http://journals.openedition.org/ceb/1484 ; DOI

https://doi.org/10.4000/ceb.1484

Ce document a été généré automatiquement le 6 juillet 2021.

\section{(c) (†) 8}

Cahiers balkaniques est mis à disposition selon les termes de la Licence Creative Commons Attribution - Pas d'Utilisation Commerciale 4.0 International. 


\title{
Écriture, histoire et action
}

\author{
Comment lire Le Derviche et la mort de Meša Selimović ? \\ Writing, History, and Action: How to Read "Death and the Dervish" by Meša \\ Selimović?
}

Branka Šarančić

1 S'il arrive fréquemment qu'un écrivain choisisse de situer son intrigue romanesque dans un passé lointain, il est bien plus rare de voir cette démarche susciter autant de polémiques que dans le cas de Meša Selimović. De toute évidence, cet auteur Bosniaque et musulman de par ses origines, mais Yougoslave et athée de par ses convictions idéologiques - fait du passé un usage qui ne rentre pas dans les schémas habituels caractérisant le genre du roman historique.

2 Avec ses romans Le Derviche et la mort (publié en 1966) et La Forteresse (1970) ${ }^{1}$, Selimović relève à son tour le défi andrićien : ressusciter par des moyens littéraires une période trouble de l'histoire nationale et collective, en rompant d'un côté avec l'image idéalisée de l'Empire ottoman, telle qu'elle était véhiculée par une littérature nationale à caractère local et, de l'autre côté, avec la tradition de la poésie populaire épique (serbe ou croate), pour qui la période ottomane figure le pire épisode du passé national. Donc, on cherchera en vain dans l'écriture de Meša Selimović cet exotisme et cet émerveillement chers à ce que l'on désigne parfois sous le nom de " roman oriental ». Les héros de Meša Selimović, dotés pour la plupart d'un bagage intellectuel bien supérieur à celui des héros de Ivo Andrić (Ahmed Nurudin, le héros-narrateur du Derviche et la mort, appartient à un des ordres religieux les plus réputés, alors que Ahmet Šabo, le chroniqueur présumé des événements décrits dans La Forteresse, est doté d'une solide éducation, y compris en matière de langues), portent un regard extrêmement lucide sur les difficultés sociales et économiques que traverse la Bosnie sous la domination ottomane. Selimović lui-même, dans ses essais critiques, souligne l'importance des auteurs qui, à l'instar de Hasan Kikić - cet autre poète désenchanté de la condition bosniaque - s'intéressent à la « vraie Bosnie », celle que l'on ne voit guère dans la littérature bosniaque de l'entre-deux-guerres, majoritairement tournée vers les thèmes idéalisés de la vie musulmane ${ }^{2}$. 
3 Mais, faut-il en conclure que Meša Selimović se fait le défenseur du «roman social », genre qui fait son apparition dans la production littéraire bosniaque pendant l'entredeux-guerres et qui, de par les services idéologiques qu'il était censé rendre dans l'émancipation de la classe paysanne, n'est pas sans faire penser à l'esthétique du « réalisme socialiste »? En fait, à se pencher de plus près sur l'essai critique que Meša Selimović consacre à l'œuvre de Kikić en $1952^{3}$, on se rend vite compte que ce dernier n'est considéré comme une figure marquante de l'histoire littéraire bosniaque que parce qu'il réussit, dans ses meilleures réalisations, à dépasser le schématisme imposé par les lois du genre et à produire une œuvre véritablement artistique. Sans négliger l'importance que revêt l'engagement politique de Kikić aux côtés des forces progressistes de la société, Meša Selimović apprécie son écriture pour ses qualités authentiquement créatrices.

Dans un certain sens, le roman sur Le Derviche et la mort, fruit d'une longue période de doutes, de tâtonnements et d'incertitudes, pendant laquelle Selimović remet en question jusqu'à sa propre capacité d'écrire, peut être considéré comme une réponse créatrice à des exigences théoriques déjà formulées dans son œuvre critique. Dans un climat littéraire hostile, où s'affrontent les défenseurs de la "littérature engagée " (pour qui l'activité artistique n'a de sens que subordonnée à un projet social et politique plus vaste) et les partisans de la «littérature pure» (que la préoccupation formelle rend insoucieux de la réalité sociale et politique), Selimović fait figure d'exception : son écriture prétend à redéfinir le rapport même entre la littérature et la politique en attribuant à cette première des pouvoirs inédits.

5 À la fois homme d'action, ayant pris une part active dans les activités révolutionnaires pendant la Seconde Guerre mondiale, et homme de contemplation, ayant un goût avéré pour les études de lettres ${ }^{4}$, Selimović était amené, de par la nature ambivalente de son rapport au monde, à prendre conscience des limites inhérentes à chacune des deux approches du réel. À travers l'histoire tragique du derviche Nurudin (que l'on voit obligé, suite à l'assassinat injustifié de son frère, de quitter le monde protégé de ses croyances religieuses pour affronter la dure réalité, avec tous les rapports sociaux et politiques pervers qui la caractérisent), on dirait que Meša Selimović prend position dans les polémiques littéraires et idéologiques qui bouleversent à l'époque les milieux intellectuels et politiques. En ce sens, ses recours aux procédés narratifs caractéristiques de la littérature moderne - récit à la première personne, renversement de l'ordre chronologique, effacement du narrateur omniscient, perspectivisme des points de vue, utilisation des techniques de «monologue intérieur » et de « courant de conscience» - alors que son intrigue a pour cadre le décor archaïque de l'Empire ottoman, posent une série de problèmes devant la critique littéraire traditionnelle, appréciant les classements distincts et les catégories stables.

6 Comment aborder Le Derviche et la mort et La Forteresse? Sous un angle plutôt esthétique ou idéologique? Qu'en est-il de leur appartenance de genre? S'agit-il du bon vieux roman historique, bien que quelque peu "rafraîchi", ou du genre entièrement moderne de roman politique, tel qu'il a été inauguré par les œuvres de Camus, de Kafka et de leurs semblables ${ }^{5}$ ? Sans vouloir entrer dans la polémique sur l'appartenance ou non des romans de Selimović à un genre littéraire préalablement identifié, il nous semble que cette indétermination même est significative du rôle que Selimović assigne à l'écriture en tant que moyen d'action idéologique. En effet, même s'il est tout à fait possible de dégager dans l'œuvre de Meša Selimović (ainsi que dans toute autre œuvre 
qui prétend à une lecture critique de la réalité) des éléments d'une vision politique, notre intérêt de chercheur va plutôt à ce qui figure l'articulation la plus intime du littéraire et de l'idéologique, c'est-à-dire sa philosophie romanesque. Car notre but n'est pas de dresser le portrait idéologique de l'auteur réel, mais d'interroger l'identité sociale, nationale et intellectuelle de la figure symbolique de l'écrivain que son écriture contribue à construire ${ }^{6}$. À ce propos, il faut préciser que notre démarche critique ne s'appuie pas sur la théorie du «reflet», selon laquelle la littérature traduirait fidèlement les éléments de la réalité extérieure, mais sur la théorie du "prisme», ou encore celle de la "trace », pour qui les images littéraires ont besoin d'être décryptées et réinterprétées?

7 Donc, en ce qui concerne l'image de la période ottomane dans l'œuvre de Meša Selimović, il ne s'agit pas pour nous de nous prononcer sur son éventuelle authenticité, encore moins sur sa valeur soit "négative» soit "positive »". Ce qui nous intéresse n'est pas la conformité de cette image à une réalité extérieure, mais l'usage que Selimović en fait dans le cadre de son économie narrative. Il n'est pas inutile de rappeler que Meša Selimović lui-même a toujours refusé pour ses œuvres l'étiquette de « romans historiques" en objectant que, si le cadre oriental dans lequel s'inscrit son intrigue romanesque est historiquement daté, les problèmes et les interrogations que soulève son écriture ne le sont pas pour autant.

Bon élève d'Ivo Andrić', Selimović est conscient que ce qui se joue dans les débats sur la transposition romanesque de la réalité dépasse de loin le domaine de l'« esthétique pure ». En se réclamant du droit de l'écrivain à une lecture tout à fait personnelle et originale du passé national, il vise à renégocier les rapports mêmes entre le littéraire et le politique. Ainsi - et c'est en cela que les œuvres d'Andrić et de Selimović occupent une place unique dans les littératures balkaniques, - l'image de l'Empire ottoman devient-elle un enjeu dans la défense de l'indépendance idéologique de l'artiste. Ce qui intéresse plus particulièrement notre propos d'aujourd'hui est justement la façon dont les innovations sur le plan littéraire permettent des innovations sur le plan des représentations identitaires. Autrement dit, nous voudrions savoir par quels moyens narratifs et grâce à quelles opérations spécifiques d'écriture la modernité revendiquée sur le plan esthétique permet de construire et faire vivre une position idéologique inédite.

9 À part l'usage des techniques narratives spécifiques que nous avons déjà relevé, une des manifestations importantes de la modernité dans l'œuvre de Meša Selimović concerne sa manière de mobiliser les références au Coran. En fait, cela est déjà assez connu, chacun des seize chapitres qui composent Le Derviche et la mort commence avec un verset tiré du livre sacré. En revanche, on ignore généralement que ces quelques phrases reproduites en exergue du texte romanesque ne sont pas des citations fidèles, mais des traductions approximatives que Selimović adaptait lui-même en fonction de ses besoins narratifs et en suivant la logique interne de son œuvre. Ainsi, la fameuse épigraphe déclarant en substance que chaque être humain travaille fatalement à sa perte, ne prend tout son sens que complétée par la suite du texte sacré, que Selimović ne reproduit pas, alors qu'elle atténue l'affirmation précédente de manière significative. En effet, dans le texte original du Coran, sont exceptés de cette règle fatale de "perte» les vrais croyants, «ceux qui croient et font œuvre bonne, qui recommandent aux autres la vérité et la patience $»^{10}$. 

islamique, qui ont crié à la trahison de l'esprit authentique du Coran ${ }^{11}$. Mais, si ce type de réaction était en quelque sorte prévisible (il n'est pas nécessaire d'être un spécialiste de l'islam pour savoir qu'il n'est pas dans la nature des écrits saints de laisser l'homme dans le doute et dans le non-sens), ce qui étonne beaucoup plus dans la réception du Derviche et la mort c'est une lecture diamétralement opposée, qui réduit la création romanesque de Selimović à une adaptation littéraire de la philosophie orientale, ou même à une interprétation inspirée des vérités du Coran. Cette réception paradoxale de l'œuvre de Meša Selimović, conséquence d'une approche réductrice et idéologiquement partiale des faits littéraires, n'est pas sans rappeler la réception problématique des romans d'Ivo Andrić, eux aussi souvent jugés au nom d'une vision nationaliste de l'histoire collective ${ }^{12}$. En ce sens, tout comme Andrić a souvent insisté sur le fait que la création littéraire repose sur une démarche intellectuelle qui n'a rien à voir avec la démarche de l'histoire positive, Selimović a toujours revendiqué le droit de l'artiste à s'attaquer à toutes sortes de dogmes, qu'ils soient de nature politique ou religieuse. Donc, ni propagateur de la religion islamique, ni son adversaire, Selimović se sert des passages du Coran pour brosser les contours du paysage moral et spirituel dans lequel évolue son héros principal, dévoué corps et âme à sa pratique religieuse et certain du salut qui attend au bout du chemin chaque vrai croyant.

drame du Derviche et la mort commence au moment où ce héros, nommé Ahmed Nurudin, ne trouve plus dans le dogme religieux les cadres explicatifs qui lui permettraient de comprendre, sinon de déjouer, la tragédie qui lui arrive. Alors que son frère Harun est enfermé dans la forteresse (lieu hautement symbolique, où finissent tous les adversaires politiques du régime), pour s'être rendu accidentellement témoin d'une intrigue politique, le derviche - qui ne connaît pas encore les raisons de cet emprisonnement - se pose des questions sur la culpabilité du frère et sur son propre devoir de réagir. En fait, le début du roman coïncide avec la naissance du doute chez Nurudin qui, sous sa carapace de derviche, se découvre tout d'un coup bien plus « humain » et vulnérable qu'il ne l'aurait cru. Cette rencontre avec lui-même, tel qu'il ne s'était jamais soupçonné auparavant, est vécue par le derviche comme une véritable crise identitaire (d'autant plus symbolique que le nom de Nurudin signifie dans la traduction littérale « lumière de la foi ») :

«Mon nom est Ahmed Nurudin; on me l'a donné et je l'ai pris comme il m'était offert, avec fierté; mais maintenant, après toutes ces années, collées à moi comme une peau, je pense à lui avec étonnement, avec ironie aussi parfois, car être la "lumière de la foi ", je n'en ai jamais tiré orgueil et il m'arrive d'en même éprouver un certain malaise. Quelle lumière suis-je donc? Qui m'éclaire? Le Livre d'en haut? [le Coran, NDT] Un cœur pur? La droite voie? [ou la vraie direction, celle qui vient de Dieu. Coran, II, 181, NDT] L'absence de doute? Tout est remis en question et à présent je ne suis qu'Ahmed: ni cheikh, ni Nurudin. L'enveloppe tombe comme un vêtement, comme une armure; il ne reste que l'être nu, comme au premier jour $»^{13}$.

12 Ce passage est significatif de la façon dont Selimović bâtit la plupart de ses personnages, sans avoir recours à la description physique ni à la psychologie traditionnelle. Tout ce que l'on sait sur Ahmed Nurudin c'est qu'il est derviche appartenant à une confrérie réputée et que, au moment des événements, il a quarante ans (cet âge ingrat où, selon Selimović, « l'homme est encore assez jeune pour avoir des désirs, trop vieux déjà pour les réaliser $\aleph^{14}$ ). 
Les épisodes qui suivent trouvent leur unité narrative moins dans le développement de l'intrigue (pourtant digne des meilleurs scénarios de romans policiers ${ }^{15}$ ) que dans la dramatisation de ce conflit intérieur qui va finir par détruire l'univers moral et affectif de Nurudin. C'est grâce à ce mariage parfait de la tension narrative et des développements méditatifs que Meša Selimović s'affirme définitivement comme un grand romancier. En effet, ses analyses réflexives ne sont jamais données "gratuitement" (comme souvent dans ce qu'on appelle d'ordinaire le «roman moderne »), mais font partie intégrante de l'économie narrative de l'œuvre. Ainsi, le conflit de conscience qui brise Ahmed Nurudin, - et c'est pourquoi il apparaît aussi crédible aux yeux des lecteurs, - est présenté moins comme une crise intellectuelle que comme une lutte pour la survie. Dans le contexte des rapports politiques dépravés, où l'idée de justice n'est qu'une couverture pour la loi du plus fort, chaque geste se mesure et chaque mauvaise parole se paie cher. Au moment où l'espace se resserre dangereusement autour de lui, en rendant sa situation existentielle sans issue, Ahmed Nurudin s'accroche à sa pensée comme à son dernier recours. Néanmoins, il faut se méfier des passages pleins de poésie, dans lesquels Meša Selimović établit une sorte de correspondance tacite entre les états d'âme du derviche et les transformations du paysage environnant. Avec Selimović, on est loin de la mystique romantique, et toute connivence entre l'homme et la nature est gratuite, même celle qui va dans le sens négatif, comme c'est le cas dans le passage qui suit:

"Âme morne de la montagne, l'ombre de l'après-midi s'étirait, rampait sur la plaine et l'assombrissait, elle s'étendit aussi sur moi, m'entoura de tous côtés, tandis que la partie ensoleillée fuyait devant elle, refluait vers un autre mont. La nuit est loin, ceci n'est que son signe avant-coureur, il y a quelque chose de sinistre dans cette obscurité qui la précède. Personne dans la plaine que l'ombre divise, je suis seul dans cet espace disputé, qui s'obscurcit, s'amenuise, se referme, seul en proie aux troubles angoisses que porte cette âme, séculaire, étrangère et pourtant mienne. Seul dans la plaine, seul au monde, impuissant en face des secrets de la terre et de l'immensité du ciel $»^{16}$.

14 Le derviche, partagé entre les deux royaumes (céleste et terrestre), ne peut plus compter ni sur la consolation de la foi ni sur la compassion des hommes. Ce sentiment d'encerclement et de solitude existentielle est également abordé dans La Forteresse, roman qui poursuit la réflexion de Selimović sur les pièges qui menacent l'homme à la recherche d'une voie authentique. Cet homme s'appelle cette fois-ci Ahmet Šabo et il n'appartient à aucune confrérie religieuse. Très tôt frappé par les injustices de la vie, il fait partie de cette armée de gens anonymes que l'Empire ottoman envoie à la guerre pour consolider ses frontières extérieures (il s'agit en l'occurrence des frontières avec la Russie). L'histoire commence au moment où Ahmet Šabo, après avoir survécu aux horreurs d'une guerre aussi vaine que cruelle, se voit obligé une fois rentré chez lui à Sarajevo - de se confronter à un monde autrement absurde, régi par des lois sociales et politiques autrement cruelles ${ }^{17}$. L'attitude de révolte qu'Ahmet Šabo finira par adopter face à une société qui se complaît dans de fausses valeurs a ceci en commun avec la révolte d'Ahmed Nurudin qu'elle ne trouve pas sa justification dans une idéologie quelconque, mais dans l'instinct de survie. À l'instar du derviche, que rien ne prédestinait à entrer en conflit avec soi-même et avec le monde, Šabo lui aussi est un « révolté malgré lui » qui, s'il n'y avait pas eu l'expérience de la guerre, n'aurait probablement jamais pris le chemin de la révolte.

Pourquoi Meša Selimović a-t-il choisi de situer ses héros dans un monde aussi aliéné et aussi hostile à tout ce qui est authentiquement humain? Là encore, les réponses des 
interprètes «éclairés » ne se sont pas fait attendre. Selon les uns, l'œuvre de Meša Selimović dénonce - à tort ou à raison d'ailleurs - les abus propres au système politique corrompu de l'Empire ottoman. Selon les autres, la manœuvre idéologique de Selimović serait encore plus subtile, car derrière les images da la Bosnie ottomane celui-ci viserait en fait les aberrations du système communiste, tel qu'il était incarné en Yougoslavie titiste. Cette dernière interprétation est souvent appuyée par une donnée biographique, Meša Selimović ayant lui-même eu le malheur de perdre son frère dans des circonstances politiques spécifiques ${ }^{18}$.

Sans vouloir rentrer dans de vaines polémiques sur les « vraies intentions » de l'auteur, il nous semble plus utile de renverser la question en se demandant comment il est possible qu'une même œuvre inspire des interprétations aussi divergentes. Car le fait même que les rapports politiques décrits dans Le Derviche et la Mort et La Forteresse puissent être identifiés à des réalités historiques aussi éloignées, témoigne mieux que tout du génie romanesque de Selimović, capable de dégager du général dans le particulier et du continu dans le discontinu. Meša Selimović l'a d'ailleurs très bien dit lui-même en soulignant que le dogmatisme idéologique que combattent ses romans n'est ni celui d'hier ni celui d'aujourd'hui, mais celui de toujours (tel qu'il a déjà existé et tel qu'il existera sans doute jusqu'à la fin des jours) ${ }^{19}$. Donc, nous ne saurions approuver ni ces lectures trop «idéologisantes » qui, - surtout après la chute des régimes communistes, - deviennent presque un phénomène de mode, ni ces lectures " historicisantes », qui flattent la fibre des historiographies nationalistes (les unes et les autres nous paraissant également inaptes à rendre compte de la complexité des faits littéraires, du moins lorsqu'il s'agit des meilleures créations romanesques). Ce en quoi Meša Selimović nous semble un digne successeur d'Ivo Andrić est justement sa conception très moderne de la création artistique, qui vise à émanciper celle-ci de toutes les instrumentalisations idéologiques.

17 À ce propos, il n'est pas indifférent que Selimović ait choisi d'écrire ses deux grands romans à la première personne en déléguant sa parole aux narrateurs fictifs, censés tenir une sorte de journal intime, qui soit à la fois une chronique des événements politiques. Bien que Le Derviche et la mort privilégie le ton confessionnel, alors que La Forteresse est plus proche d'une chronique proprement dite, l'écriture à la première personne permet à Selimović de ne pas identifier ses personnages en termes d'appartenance idéologique. Ainsi, Ahmed Nurudin et Ahmet Šabo, comme nous l'avons déjà souligné, n'ont aucune ambition de nature politique, sinon de rester en paix avec le monde et avec eux-mêmes. Pourtant, et c'est là que Selimović montre à la fois la force de son talent romanesque et son sens profond du politique, ce sont les événements extérieurs, indépendants de la volonté des personnages concernés, qui déclenchent leur descente irréversible en enfer. On ne peut pas, en lisant Selimović, ne pas penser à La Cour maudite d'Ivo Andrić, elle aussi métaphore d'un huis clos existentiel, où il n'y a pas d'innocents et où les gens naissent coupables.

En choisissant comme protagonistes de ses intrigues des personnages qui présentent tous les traits des antihéros (ils sont maladroits, rancuniers, par moment même lâches ou égoïstes; ils manquent de courage et d'assurance ; ils doutent, ils se trompent et ils sont capables d'agir mal), Selimović menace dangereusement le pacte traditionnel, implicitement convenu entre un auteur et ses lecteurs. Ces derniers, habitués à s'identifier au personnage du héros principal (cette identification est d'autant plus facile lorsque celui-ci est présenté sous l'aspect de la victime, comme c'est le cas du 
derviche au début du roman), ne peuvent qu'être déconcertés par la dégradation morale que subit le derviche après s'être emparé à son tour d'un poste de pouvoir. Une telle évolution d'Ahmed Nurudin, inattendue du point de vue des attentes morales du lecteur, mais rendue logique par le développement narratif de l'intrigue, atteint son point culminant au moment où le derviche, devenu entre-temps cadi ${ }^{20}$, se trouve amené à trahir plus que son meilleur ami - son frère spirituel, Hasan. Ce faisant, le derviche, en toute conscience, cautionne les mêmes comportements politiques qui avaient jadis coûté la vie à Harun, son frère de sang. Ainsi le roman se clôt par le désarroi le plus complet du héros principal qui, ayant perdu sa foi en la justice de Dieu et en celle des hommes, attend l'arrivée de la mort (en fait de ceux qui sont désignés pour la lui donner à l'issue d'une nouvelle intrigue politique) comme son ultime délivrance. Fidèle à lui-même jusqu'aux dernières pages du roman, Selimović problématise le sens, ou plutôt le non-sens de cette ultime étape, qui s'avère aussi incompréhensible et difficile à assumer que la vie elle-même :

"Assis sur mes talons, j'écoute. Dans le silence de la chambre, l'horloge invisible bat, dans un espace invisible, la marche irrépressible du destin.

L'effroi me submerge, tel un flot.

Les vivants ne savent rien. Apprenez-moi, ô morts, à mourir sans effroi, du moins sans horreur. Car la mort est un non-sens, comme la vie $»^{21}$.

Quelle pourrait être la morale à tirer de cet étrange roman qui se propose d'éclairer la vie et la mort d'un derviche, l'une aussi absurde que l'autre? Même s'il ne la formule pas en ces termes, c'est la question que le lecteur traditionnel risque facilement de se poser après la lecture du roman. Sous ses apparences anodines, cette remarque soulève d'importantes interrogations sur le plan théorique : en effet, elle réactualise un autre débat, implicitement contenu dans toutes les querelles littéraires - celui qui porte sur la finalité même de la littérature. Car, s'il n'y a aucune morale à tirer et aucun message lisible à capter, à quoi bon lire les œuvres romanesques (si ce n'est pour s'adonner au seul plaisir de suivre les rebondissements de l'intrigue)?

Quand il s'agit de Meša Selimović, même une lecture peu attentive suffit pour se rendre compte de l'absence totale de prétention moralisatrice dans son œuvre. Le parcours surprenant du héros-narrateur qui, d'ancienne victime, se transforme en bourreau malgré lui, non seulement rompt avec une vision idéalisée de la victime, mais aussi éclaire d'un jour différent - voire humanise - la position du bourreau. En fait, l'univers d'angoisse et d'oppression (lorsque les rapports de domination sont à ce point dépouillés de toute justification raisonnée qu'on désigne d'habitude sous le nom d'« idéologie », on a du mal à parler de "système politique »), dans lequel évoluent les héros de Selimović, a ceci de particulier qu'il s'avère également avilissant et aliénant pour les oppresseurs que pour les opprimés. Ahmed Nurudin, lorsqu'il se retrouve de l'autre côté du miroir, paye cette découverte de sa propre tête ${ }^{22}$.

On l'aura bien compris : avec Le Derviche et la mort, nous sommes aux antipodes du roman à thèse. L'auteur omniscient s'efface devant une multiplication des points de vue. Il n'y a plus de cette autorité auctoriale rassurante qui, cautionnant un système de valeurs cohérent, garantit l'unité du monde décrit. On aura beau chercher dans l'œuvre de Selimović les réponses toutes faites et les vérités définitives. Les catégories du bien et du mal y sont toutes relatives, sinon étroitement unies. Le sentiment de confusion que peut éprouver un lecteur non averti face à ce jeu subtil des points de vue, à ces incessants changements de perspective, est renforcé par l'usage très particulier que Meša Selimović fait du monologue intérieur ${ }^{23}$. Celui-ci est tantôt assumé par le 
personnage du derviche, qui vit ses propres doutes en temps réel, tantôt par le personnage du narrateur, qui revit les épisodes de sa vie en les écrivant (et dont les commentaires viennent compléter, corriger ou contredire cette "vision en temps réel »), tantôt par Selimović lui-même, qui intervient en tant qu'instance auctoriale indépendante (en brouillant plus encore les schémas perceptifs).

En quelque sorte, les errances spirituelles du derviche ne font qu'un avec la structure polyphonique du roman : les voix et les interrogations se multiplient sans que l'on soit pour autant plus près de la réponse. Et comme si cette complication n'était pas encore suffisante, Selimović n'établit pas de frontière nette entre ses personnages. Ainsi, Hasan ne joue pas seulement le rôle du meilleur ami d'Ahmed Nurudin (que celui-ci finit par trahir), mais incarne aussi la version plus humaine du derviche, l'homme que celui-ci aurait pu devenir dans une autre vie. Il en est de même avec le personnage imaginaire d'Ishak, dont les apparitions fantasmagoriques coïncident avec les moments de désarroi culminants de la crise identitaire du derviche. Ishak, doté de tous les attributs qui caractérisent un rebelle de nature (courage, audace, décision, mépris de la mort...), figure lui aussi une réalisation virtuelle du personnage du derviche : il est ce double si envié, qui échapperait au conditionnement psychique du commun des mortels. Ainsi, paradoxalement, s'il fallait chercher à identifier le personnage porteparole de l'auteur, on serait dans l'obligation d'admettre que c'est à la fois chacun et personne, car c'est la pluralité même des points de vue qui traduit la position idéologique de Selimović.

Si les romans de Selimović sont aux antipodes du roman à thèse, ils sont tout aussi éloignés du roman d'apprentissage. Même si apprentissage il y a, il est conçu plutôt dans un sens négatif: plus on découvre la réalité de la vie et celle des rapports humains, moins on est capable d'y voir clair. Apprendre dans le schéma cognitif de Selimović signifie renoncer à ce que l'on sait déjà, ou plutôt, à ce que l'on croit savoir. L'immensité du désarroi dans lequel sombre le derviche à la fin du roman est à l'image de sa foi initiale : c'est le prix qu'il doit payer pour accéder à la connaissance. Ce n'est qu'une fois fait le deuil de son ancien moi (le moi du derviche) qu'Ahmed Nurudin peut commencer le dur apprentissage :

"Je suis derviche depuis vingt ans. Petit enfant, je suis allé à l'école, et je ne sais rien à part ce qu'on a voulu m'apprendre. On m'a appris à écouter, à souffrir, à vivre pour la foi. Il y en a eu de meilleurs que moi, de plus fidèles, pas beaucoup. J'ai toujours su ce qu'il fallait faire, l'ordre des derviches pensait pour moi, et les bases de la foi sont solides et larges. Il n'existait rien en moi qui ne pût s'y conformer ${ }^{24}$.

Le passage cité, on l'aura bien compris, ne parle pas que de la foi religieuse, mais de toute autre croyance qui prend la forme de dogmatisme idéologique. À travers l'histoire tragique du derviche, Selimović dénonce ce penchant naturel qu'ont les hommes à se cacher derrière les vérités consensuelles, entérinées par une autorité supérieure (qu'il s'agisse du dogme religieux, de la doxa politique ou des préceptes de la tradition et de la morale conventionnelle), en évitant d'élucider par eux-mêmes les choses qui leur arrivent. Néanmoins, s'agissant de cette exigence spirituelle que formule Selimović, et qui consiste à dépasser le conditionnement de l'expérience, de l'éducation et de l'habitude, une question s'impose d'emblée : quel est cet autre point de vue qu'il faudrait adopter pour échapper aux préjugés de la morale, de la religion et de la politique ? Le sort tragique que subit le derviche à la fin du roman ne suffit-il pas en lui-même pour suggérer l'absurde de toute tentative de résistance dans un monde aliéné où aucun compromis n'est plus possible entre l'individu et la société, entre les 
valeurs spirituelles et les principes d'action politique ? Existe-t-il encore un espace de liberté intellectuelle et d'engagement authentique?

Tant que l'on partage le point de vue du personnage romanesque du derviche, la réponse est bien négative. Ahmed Nurudin ne trouve aucun réconfort ni dans la méditation religieuse ni dans le champ d'action politique. En échangeant sa condition de derviche contre celle de cadi il se rend compte que le royaume céleste n'a rien à envier au pouvoir terrestre, les deux étant également inappropriés aux aspirations les plus profondes de l'homme. Pourtant, les choses se présentent sous un tout autre angle vues du point de vue du narrateur (instance fictive qui assume l'écriture du récit). Celui-ci n'agit plus ni au nom des valeurs spirituelles prônées par l'ordre des derviches, ni au nom de la vengeance politique qui oriente les actes du cadi. Pour lui, l'espace de l'écriture figure sa dernière chance de récupérer l'irrécupérable, c'est-à-dire de s'assumer en tant qu'individu libre de toutes les pressions de l'action immédiate et débarrassé de toutes les idéologies. Il est tout à fait extraordinaire de voir comment Selimović place des idées extrêmement modernes sur le sens et la finalité de la littérature dans un passage à l'allure anodine, où le narrateur s'étonne lui-même de l'effet produit par son écriture :

«Au fait, pourquoi ai-je écrit révolte! C'est la première fois que ce mot me vient à l'esprit; mon tourment, dont j'ai pris conscience depuis peu, pour moi, portait un autre nom. Révolte! d'où vient ce mot dangereux? Je me demande s'il ne vaudrait pas mieux cesser d'écrire afin de ne pas aggraver les choses. Car si par des voies inexplicables, je tire de moi-même ce que je n'avais pas l'intention de dire, ce qui ne répond pas à ma pensée, ou qui est ma pensée ignorée, dissimulée dans le tréfonds obscur de mon être, saisi par une émotion que je ne contrôle plus, s'il en est ainsi il faut cesser cette enquête implacable, cette tâche diabolique, briser cette plume de roseau, répandre l'encre sur le seuil de pierre de la tekké25, et que cette tache noire me détourne à tout jamais de ce maléfice. Révolte! N'est-ce qu'un mot, ou vraiment une pensée? Si c'est une pensée, alors c'est ma pensée, ou mon égarement. Si c'est de l'égarement, malheur à moi, mais si c'est la vérité, il faut craindre le pire $»^{26}$.

Ces « voies inexplicables » par lesquelles le narrateur tire de lui-même ce qu'il n'avait pas l'intention de dire, cette « enquête implacable » que l'alchimie incontrôlée des mots apparente à une "tâche diabolique ", c'est bien l'écriture en action, telle qu'elle permet d'éclairer les choses sous un angle inédit. Là où abdiquent le derviche et le cadi, persévère le narrateur à la recherche d'un sens à donner à la tragédie qu'il est en train de vivre. Ainsi, sous l'habit désuet du derviche, le lecteur découvre une figure extrêmement moderne de l'écrivain, qui n'a rien à envier à la philosophie romanesque $\mathrm{du}$ narrateur proustien. Ce qui rapproche les deux représentations symboliques $d u$ littéraire c'est justement l'idée que la posture de radicalisme spirituel, inhérente à l'action d'écrire, permet de déjouer les mécanismes de conditionnement psychologique et idéologique, et d'accéder ainsi à une nouvelle liberté intellectuelle. Ce n'est pas un hasard si pour les deux narrateurs (celui du Derviche et la mort et celui de la Recherche du temps perdu) le début de l'écriture coïncide avec la fin de la vie : tout se passe comme si l'imminence de la mort était le meilleur gage de la véridicité de leur discours. C'est parce qu'il n'a plus rien à attendre des vivants (tous les liens sociaux et affectifs sont désormais coupés avec le monde environnant et il n'y a plus aucune possibilité de salut) que le derviche se tourne vers les morts, dans une sorte de dialogue muet qui n'a d'autre finalité que d'entretenir le fil menu du récit.

27 Quel intérêt d'aborder la question de la modernité de la philosophie romanesque de Selimović lorsqu'il s'agit d'interroger l'image de la période ottomane dans son œuvre? $\mathrm{Au}$ risque de paraître trop « littéraire » dans notre démarche, il nous semble que la 
complexité du point de vue de Selimović impose une extrême prudence quand il s'agit d'interpréter les images parfois contradictoires qu'il associe à la Bosnie ottomane. Comment choisir celle qui serait représentative de la position idéologique de l'auteur? La "vraie Bosnie» serait-ce ce pays mal gouverné, en proie au despotisme des puissants locaux, aussi stupides que cruels et corrompus, ce régime de terreur et de police que décrit une lettre suspecte, interceptée par le toujours vigilant Piri-Vojvoda, aux aguets de nouvelles affaires d'espionnage, et écrite de la main d'un commerçant de Dubrovnik, soupçonné d'espionnage ${ }^{27}$ ? Ou peut-être ce pays de misère morale et matérielle, où tout n'est que férocité, malheur, souffrance, privation..., pays où la méchanceté et la barbarie des gens n'ont d'égales que l'hypocrisie derrière laquelle ils se cachent (tout ce contre quoi se révolte le personnage de La Forteresse, Šehaga Sočo ${ }^{28}$, dont le fils est liquidé comme déserteur, alors qu'il s'était engagé à la guerre comme volontaire). Serait-ce au contraire ce pays rêvé et jamais oublié que Ferhad, personnage cousin du narrateur de La Forteresse, vient retrouver après vingt années d'exil en payant cet amour sans réserve de sa propre tête ${ }^{29}$ ? Ou encore, cette terre d'une beauté inégalée, dont les magnifiques paysages résonnent dans l'âme tourmentée du derviche?

Il serait impossible, voire inutile de relever toutes les images que les personnages de Selimović attribuent à la Bosnie de la période ottomane. Celle-ci est parfois l'objet d'un amour inconditionnel, et parfois d'une haine sans merci. Pourtant, ce qui est caractéristique de la démarche narrative de Selimović, c'est que ces images n'ont pas une valeur «absolue ", mais sont motivées par l'histoire personnelle des protagonistes. Ainsi, au moment où Šehaga s'apprête à mourir dans des circonstances mal éclaircies, Selimović relativise la portée de sa révolte à l'égard de la Bosnie, en suggérant que cette haine était aussi pour Šehaga une manière de faire face à la perte de son fils. Il en va de même avec le personnage de l'étudiant Ramiz (le seul personnage de La Forteresse qui est censé adhérer à un programme politique précis), dont les idées sur la Bosnie sont déterminées par les prérogatives de son action idéologique.

La vision de Hasan, ce double plus "humain » du derviche, mérite quant à elle plus d'attention, car elle est à la fois plus nuancée et moins dictée par le conditionnement psychologique et idéologique du personnage. Il est inoubliable, ce passage où Hasan compare la Bosnie au personnage de l'infirme Djemaïl qui, tant qu'il est assis, surprend par sa beauté et sa force, mais dès qu'il se lève, se fait remarquer par son handicap et sa laideur manifestes. À propos de ce Djemaïl, devenu infirme après s'être mutilé luimême sous l'effet de l'alcool (et qui continue, lorsqu'il a bu, à planter son couteau dans les moignons desséchés, ne permettant à personne de l'approcher), Hasan dit qu'il est l'image même de la Bosnie: "Une force sur des moignons. Son propre bourreau. Une richesse qui ne débouche sur rien $»^{30}$. Il s'en suit un dialogue entre lui et le derviche. À la question du derviche: "Que sommes-nous alors? Des fous? Des malheureux?", Hasan répond:

"Les hommes les plus compliqués qui soient au monde. L'histoire ne s'est amusée aux dépens de personne autant qu'aux nôtres. Hier encore nous étions ce que nous souhaitons oublier aujourd'hui. Mais nous ne sommes pas devenus autres. Nous nous sommes arrêtés à michemin, ébaubis. Nous n'avons plus où aller. Nous avons été déracinés, mais non accueillis. Tel un bras mort séparé du lit de la rivière par les crues, trop petit pour être un lac, trop grand pour que la terre le boive. Confusément honteux de notre origine, de notre reniement aussi, nous refusons de regarder en arrière, nous n'avons pas devant nous où poser les yeux; nous retenons alors le temps, redoutant toutes les solutions, quelles qu'elles soient. Méprisés à la fois par nos frères et par les nouveaux venus, nous brandissons pour nous défendre la 
fierté et la haine. Nous avons voulu notre intégrité, et nous l'avons perdue au point de ne plus savoir même ce que nous sommes. Le malheur est que nous nous sommes pris à aimer ce bras mort où nous flottons, et que nous ne voulons pas en sortir. Et tout se paie, même cet amour. Est-ce par hasard que nous sommes ainsi exagérément mous et exagérément rudes, sensibles et durs, gais et tristes, toujours prêts à étonner quelqu'un, nous les premiers? Estce par hasard que nous nous abritons derrière l'amour, seule certitude dans toute cette confusion, que nous laissons la vie nous écraser, que nous nous détruisons, d'une autre manière que Djemail, mais aussi sûrement? Pourquoi le faisons-nous? Parce que nous ne restons pas indifférents. C'est donc que nous sommes honnêtes, nous pouvons être fiers de notre folie $»^{31}$.

Peut-on décrire mieux que cela la complexité de l'héritage historique que représente la Bosnie de la période ottomane? Le personnage de Hasan qui, après avoir interrompu ses études à Constantinople, où il s'apprêtait à devenir quelqu'un d'important dans la hiérarchie de l'Empire, rentre en Bosnie pour devenir un simple marchand de bétail réunit en lui-même toutes les contradictions de cet impossible choix entre l'Orient et l'Occident. Ayant senti par lui-même les préjugés que les gens de Constantinople cultivaient à l'égard du peuple bosniaque, considéré comme sauvage et inculte, et la mesquinerie de ses propres compatriotes qui ne cherchaient à voir dans son retour qu'un signe certain de son échec cuisant (car «aucun homme sensé n'aurait quitté Istanbul pour cette modeste bourgade, ni renoncé à des fonctions importantes, à moins d'y être contraint $\|^{32}$ ), Hasan apprend à pardonner aux uns et aux autres. Son mérite, c'est qu'il s'efforce de ne pas succomber à une réaction affective, mais d'essayer de comprendre les raisons d'un tel état de choses. Devant les railleries que les gens d'Istanbul formulent sur le compte de son pauvre pays, cette «lointaine province perdue, arriérée », il s'exclame :

\begin{abstract}
«Nous, nous n'appartenons à personne, nous nous trouvons toujours sur une frontière ou sur une autre; nous sommes toujours la dot de quelqu'un. Est-il donc étonnant que nous soyons pauvres? Depuis des siècles nous nous cherchons, nous nous trouvons parfois. Bientôt nous ne saurons plus qui nous sommes, certains nous font l'honneur de nous ranger sous leur drapeau, puisque nous n'en possédons pas; ils nous allèchent lorsqu'ils ont besoin de nous et nous rejettent ensuite; nous sommes le vilayet le plus pitoyable au monde, les hommes les plus malheureux du monde, nous perdons peu à peu notre propre visage et ne pouvons prendre celui des autres; nous sommes déracinés et jamais accueillis, pour tous des étrangers, pour ceux de notre race comme pour ceux qui ne nous acceptent pas dans la leur. Nous vivons à la limite des mondes, à la frontière des peuples, exposés à toutes les attaques, toujours coupables. Les vagues de l'histoire se brisent sur nous, comme sur un rocher. Nous sommes las de la violence, et avons fait de notre malheur une vertu; nous sommes devenus généreux par défi. Vous, vous êtes brutaux sans motif. Qui de nous est arriéré ? "33
\end{abstract}

Cet amour-haine que Hasan porte à son pays n'est pas sans faire penser à la façon complexe dont Selimović se déterminait par rapport à ses origines ${ }^{34}$. En effet, pour Selimović, le véritable amour (qu'il s'agisse des sentiments amoureux ou des sentiments patriotiques) n'est pas celui qui est fondé sur une vision idéalisée de l'objet vénéré, mais celui qui, sans être dupe de la réalité des choses, arrive à les accepter et aimer telles quelles. L'écriture littéraire représentant aux yeux de Selimović un exercice de radicalisme spirituel par excellence, écrire sur son propre pays était pour lui bien plus qu'un devoir patriotique: un défi d'artiste ${ }^{35}$. Ce défi est d'autant plus ambitieux qu'il s'agit d'un pays dont la complexité identitaire pose toujours un problème tant qu'on l'aborde du point de vue des positions idéologiques dogmatiques (d'où les interprétations souvent contradictoires de la période ottomane dans les historiographies nationales des pays concernés). Grâce à une conception très originale de la création littéraire, Selimović se sert de son écriture comme d'un moyen à part 
entière d'action idéologique (dans le sens le plus large du mot), en léguant à ses lecteurs une image de la Bosnie ottomane, non pas telle qu'elle fut réellement, mais telle qu'elle peut être lue et comprise par un peuple à la recherche de sa propre histoire.

Pour finir, il nous semble que la création romanesque de Meša Selimović, de par les importantes innovations qu'elle introduit tant sur le plan littéraire que sur le plan identitaire, ouvre un nouveau champ de réflexion sur les rapports entre écriture, histoire et action. Car la force de persuasion avec laquelle les univers romanesques de Selimović et d'Andrić continuent à agir sur leurs lecteurs est la meilleure preuve que c'est dans l'écart entre le "vrai » et le "possible» que se situe l'espace d'action littéraire. Le fait que la période ottomane, aussi problématique fût-elle, ait pu inspirer la naissance de deux œuvres littéraires d'une telle beauté a quant à lui un impact bien réel sur la façon d'envisager l'histoire nationale et collective.

\section{NOTES}

1. Avant ces deux grands romans, qui ont consacré Selimović comme grand auteur, il avait déjà publié deux autres romans, Tišine [Silences] en 1961 et Magla i mjesečina [Brouillard et clair de lune] en 1965, plusieurs nouvelles, ainsi qu'un livre d'essais critiques.

2. À part des histoires d'amour et des récits de harem, on y retrouve notamment différentes scènes de la vie familiale des dignitaires musulmans. Voir l'essai consacré à l'œuvre de Hasan Kikić dans Meša Selimović, Pisci, mišljenja i razgovori : eseji, članci, polemike, intervjui [Écrivains, opinions et conversations : essais, articles, polémiques, interviews], pp. 35-131 (particulièrement p. 59), Sabrana dela u deset knjiga ; 8 [Euvres complètes ; t. 8], Beograd : Sarajevo, BIGZ : Svjetlost, 1990.

3. Quatorze ans avant la parution de son chef-d'œuvre, Le Derviche et la mort.

4. Né en 1910 à Tuzla (Bosnie-Herzégovine), où il a terminé son lycée, Selimović fait ses études supérieures à la Faculté de Lettres de Belgrade (département langue serbo-croate et littérature yougoslave). Avant la guerre, il a travaillé comme professeur au lycée de Tuzla. Après la libération, il passe quelques années à Belgrade, puis s'installe à Sarajevo, où il joue un rôle important dans la vie culturelle du pays (il est tour à tour Maître de conférences à la Faculté de Lettres de Sarajevo, directeur de la grande maison cinématographique "Bosna-film », directeur dramatique du Théâtre national de Sarajevo et, à partir de 1961, rédacteur en chef de la très importante maison d'édition « Svjetlost »).

5. C'est cette dernière piste qui a été retenue par Nikola Kovač dans son remarquable essai consacré aux romans Le Derviche et la mort et La Forteresse, intitulé «Politički apsurd i ljudski otpor » [Absurde politique et résistance humaine], dans Roman, istorija, politika : eseji [Roman, histoire et politique : essais], Sarajevo, « Veselin Masleša », 1988, pp. 166-203. La piste idéologique (morale et éthique) a été suivie également par Kasim Prohić, dans Činiti $i$ biti : roman Meše Selimovića [Faire et être : à propos du roman de Meša Selimović], Sarajevo, Svjetlost, 1972.

6. Cette figure symbolique, qui à la fois anticipe et programme la réception de l'œuvre, parle à sa façon de la réalité sociohistorique dans laquelle l'œuvre prend son sens. 
7. Sur la médiation du champ littéraire dans la prise de position idéologique des écrivains, on consultera utilement les travaux fondateurs de Pierre Bourdieu, Christophe Charle, Rémy Ponton, Alain Viala (s'appuyant sur des exemples de la littérature française).

8. C'est justement cette lecture directe, au premier degré, qui a valu à l'œuvre de Meša Selimović autant de polémiques. Accusé tantôt de verser trop dans la philosophie orientale et le folklorisme local, tantôt - au contraire - de profaner ses racines musulmanes en compromettant l'image de la Bosnie ottomane, Selimović s'est toujours défendu de cette identification trop rapide avec les personnages fictifs de ses romans.

9. Bien que, par modestie, il ait toujours refusé de se comparer à l'auteur du Pont sur la Drina et de La Chronique de Travnik, Meša Selimović n'a jamais caché sa grande admiration pour l'œuvre qu'il considérait comme une des meilleures réalisations des littératures ex-yougoslaves.

10. Nous nous servons ici, et cela sera le cas tout au long de notre article, de la traduction faite par Mauricette Begić et Simone Meuris, Le Derviche et la mort [titre original : Derviš i smrt], [Paris], Gallimard, coll. «L'imaginaire », 2004 (voir la NDT nº 3, p. 13).

11. Selimović se défend de ce type d'attaques dans son interview pour le journal Preporod [Renaissance] en 1972 ; voir Pisci, mišljenja i razgovori : eseji, članci, polemike, intervjui [Écrivains, opinions et conversations : essais, articles, polémiques, interviews], op. cit., p. 377.

12. Là où les uns voyaient une image mensongère et dépréciative de la période ottomane, d'autres cherchaient une vérité historique objective. Pour plus d'informations sur le sujet, voir dans ce même numéro notre article « L'Empire ottoman d'Ivo Andrić : à la recherche de l'histoire perdue $"$.

13. Le Derviche et la mort, op. cit., p. 14.

14. Ibidem.

15. Bien que très moderne dans ses conceptions de l'art romanesque, Selimović a toujours considéré qu'un bon roman ne saurait pas se passer de l'intrigue dans le sens classique du mot; voir Pisci, mišljenja i razgovori... [Écrivains, opinions et conversations...], op. cit., p. 264. Il est rare qu'un roman aussi méditatif que Le Derviche et la mort soit associé à une intrigue aussi habilement construite.

16. Le Derviche et la mort, op. cit., p. 89. Selimović dira ailleurs que la nature a un effet consolateur pour l'homme justement par ce qu'elle est indifférente à ses souffrances. Au fond, dans l'économie romanesque de Meša Selimović, les développements poétiques ne sont là que pour mieux suggérer le contraste qu'il y a entre le monde réel et celui auquel les hommes aspirent.

17. Un des personnages du roman, Mula Ibrahim, dit à propos de la guerre : «La guerre, c'est un combat cruel, mais honnête, comme la lutte des animaux. La vie dans la paix est un combat aussi cruel, mais malhonnête, comme cela est propre aux êtres humains. La différence est énorme »; voir Meša Selimović, Tvrđava, Beograd : Rijeka, «Sloboda »: «Otokar Keršovani », 1975, p. 147 (c'est nous qui traduisons), Sabrana dela ; 2 [Euvres complètes ; t. 2].

18. Celui-ci, bien que hautement placé dans la hiérarchie politique yougoslave, a été fusillé vers la fin de la guerre accusé d'avoir détourné des objets appartenant à la propriété collective.

19. Pisci, mišljenja i razgovori... [Écrivains, opinions et conversations...], op. cit., p. 348.

20. Magistrat musulman qui remplit des fonctions civiles, judiciaires et religieuses.

21. Le Derviche et la mort, op. cit., p. 375.

22. Bien sûr, ces rapports dénaturés qui régissent le monde des héros de Selimović ne sont pas sans suggérer une certaine image du fonctionnement administratif et politique de l'Empire ottoman, surtout pour ce qui est des pouvoirs quasi illimités conférés à l'individu. Néanmoins, en abordant le thème du pouvoir absolu (applicable aux tyrannies de jadis aussi bien qu'aux systèmes totalitaires d'aujourd'hui), Selimović s'intéresse moins à ses implications proprement politiques qu'aux conséquences désastreuses qu'une telle conception de la société a sur le plan des rapports humains. Là encore, en déléguant sa parole à un héros-narrateur, censé vivre tour à tour le rôle du dominé et celui du dominant, Selimović se garde de glisser dans le discours du 
« politiquement correct». La vraie tragédie du derviche, et de toute autre personne qui aurait la malchance de subir le même conditionnement politique, c'est de n'avoir que ce choix-là à faire : être parmi les bourreaux ou parmi les victimes. Néanmoins, là où Selimović laisse transparaitre comme une esquisse de sa vision politique c'est dans son idée que, dans une société où les rapports humains sont fondés sur la loi du plus fort, personne n'est gagnant, y compris ceux qui jouissent momentanément de la position de dominants (et qui sont prédestinés, de par la force des choses, à devenir eux-mêmes - tôt ou tard - victimes des nouveaux prétendants au pouvoir absolu).

23. Sur les différentes formes du monologue intérieur dans l'œuvre de Meša Selimović voir entre autres Muhsin Rizvić, «Relativizam kao struktura i poetika romana Derviš i smrt » [Relativisme en tant que structure et en tant que poétique du roman Le Derviche et la mort], dans Panorama bošnjačke književnosti [Panorama de la littérature bosniaque], Sarajevo, Ljiljan, 1994, pp. 379-387.

24. Le Derviche et la mort, op. cit., pp. 85-86.

25. Tekké ou tekkiyé, ou takiya : centre de réunion et habitation du chef, le cheikh, maison des frères de passage et surtout chapelle réservée aux exercices spirituels (NDT).

26. Le Derviche et la mort, op. cit., p. 15.

27. Sur le contenu précis de cette lettre voir Le Derviche et la mort, op. cit., p. 353.

28. Voir le passage concerné dans Tvrđava [La Forteresse], op. cit., pp. 40-41.

29. En fait, Ferhad, en train de redécouvrir les beautés de son pays, est reconnu par serdar Avdaga, enfermé dans la forteresse et liquidé au nom d'une faute qu'il aurait commise dans sa jeunesse.

30. Le Derviche et la mort, op. cit., pp. 336-337.

31. Ibidem.

32. Ibidem, p. 276.

33. Ibid., p. 272.

34. Voir sur le sujet Pisci, mišljenja i razgovori... [Écrivains, opinions et conversations...], op. cit., pp. 301-302.

35. Selon Selimović, rien n'est plus difficile pour un écrivain que d'être objectif à l'égard de sa mère et à l'égard de son pays; voir Pisci, mišljenja $i$ razgovori... [Écrivains, opinions et conversations...], op. cit., p. 293.

\section{RÉSUMÉS}

Le roman Le Derviche et la mort de Meša Selimović fait partie de ces œuvres emblématiques qui non seulement échappent à la codification traditionnelle des genres littéraires, mais contribuent à redéfinir le statut même de la littérature en tant que moyen autonome d'action idéologique. Lu tantôt comme un "roman historique ", dont l'intrigue emprunte un cadre historiquement daté (celui de la période ottomane), tantôt comme un "roman politique », continuant la lignée de Kafka et de Camus, Le Derviche et la mort est avant tout une plaidoirie pour l'indépendance idéologique de l'artiste. Publié dans un climat littéraire conflictuel, où s'affrontent les défenseurs de la « littérature engagée » (pour qui l'activité artistique n'a de sens qu'étant subordonnée à un projet social et politique plus vaste) et les partisans de la « littérature pure » (qui, préoccupés de la forme, ne se soucient guère de la réalité sociale et politique), le roman de M. Selimović vise à redéfinir le rapport même entre la littérature et la politique en proposant des solutions 
idéologiques inédites sur le plan de la création littéraire aussi bien que sur le plan des représentations identitaires.

The novel "Death and the Dervish" is part of those emblematical works which not only escape the traditional codification of the novelistic literary genres but help as well redefine the status itself of literature as an independant means of ideological action. Sometimes read as a historical novel, which plot can be historicaly identified (the Ottoman period), other times read as a political novel, in the tradition of Kafka or Camus, "Death and the Dervish" is, in the first place, a speech for the defence of artists' ideological independance. Published in a climate of conflict, where the upholders of the "engaged litterature" (those ones for whom the artistic activities have no sense if they are not subject to some vast social and political project) and supporters of the "pure literature" (those ones who, being concerned about the form, are made heedless of social and political reality) clash, Meša Selimović's novel aims at redefining the relationship itself between literature and politics by proposing original ideological solutions in both the field of literary creation and of identities representations.

\section{INDEX}

Mots-clés : littérature et politique, Derviche, Andrić Ivo (1892-1975), littérature bosniaque, Meša Selimović (1910-1982), Selimović Meša (1910-1982), Ottomans dans la littérature

Thèmes : Littérature

motsclesmk БоснА

motsclestr Bosna

Index géographique : Bosnie

Index chronologique : vingtième siècle

motsclesel Boovía

Keywords : Literature, Selimović Meša (1910-1982), Bosnia, Bosnian literature, Ottoman, twentieth century 